\title{
A call for evidence-based quality measures: a goal worthy of pursuit
}

\author{
Daniel H Solomon and Sherine E Gabriel
}

The Centers for Medicare and Medicaid Services has unveiled a program of enhanced reimbursement for doctors who report on a limited number of performance measures (http://www.cms. hhs.gov/PQRI/35_2008PQRIInformation.asp, accessed April 30, 2007). Although this program only includes 74 performance measures (of which five apply to osteoporosis and none yet to arthritis), it could represent the beginning of a trend whereby insurers require physicians to report on quality measures-which could lead to wide-scale adoption of 'pay for performance'. At this critical juncture in the development of such quality measure-based reimbursement programs, it is worth examining quality measures in rheumatology and whether they will actually enhance patient outcomes.

In the field of rheumatology, we have our own set of quality measures (MacLean $\mathrm{CH}$ et al. [2004] Arthritis Rheum 51: 193-202). These were developed using evidence-based methods and a rigorous consensus process as part of the Arthritis Foundation's Quality Indicator Project (AFQuIP). From this initial set of nearly 100 measures, the American College of Rheumatology (ACR) has adopted 16 pertaining to rheumatoid arthritis, gout, osteoporosis and laboratory monitoring. These measures focus on the process of care for rheumatic conditions (i.e. how a physician who cares for a specific patient should respond in a given situation). When these measures were originally developed in 2002 as part of AFQuIP, solid trial-based data to support such a quality measure were not available. Since then, however, the Tight Control for Rheumatoid Arthritis (TICORA) trial has clearly demonstrated that prompt titration of medications leads to improved outcomes (Grigor C et al. [2004] Lancet 364: 263-269).

The link with patient outcomes has not been established for many quality measures. The ACR drug monitoring recommendations are
How do we get from our current state of 'immature' quality measures to a set of measures that we know improve patient outcomes?

DH Solomon is an Associate Professor of Medicine and is Chief of the Section of Clinical Sciences in the Division of Rheumatology at the Brigham and Women's Hospital at Harvard Medical School in Boston, MA, USA. SE Gabriel is a member of the Nature Clinical Practice Rheumatology Advisory Board and is Professor of Medicine and of Epidemiology, and the Chair of the Department of Health Sciences Research, at the Mayo Clinic, Rochester, MN, USA.

\section{Competing interests} The authors declared they have no competing interests.

www.nature.com/clinicalpractice doi:10.1038/ncprheum0536 based on a solid foundation, but no one has ever tested whether less-frequent monitoring leads to worse patient outcomes. Regular monitoring will lead to less adverse events, but the optimum monitoring strategy for a given patient is not yet known. Quality measures from other clinical disciplines have been tested against actual patient outcomes, sometimes with surprising findings. For example, a study of congestive heart failure found that patients discharged from hospitals that provided appropriate treatments upon discharge did not experience decreased 30-day mortality rates. This sort of finding should cause us to pause and consider how to improve the quality measures that physicians will be asked to report on.

How do we get from our current state of 'immature' quality measures to a set of measures that we know improve patient outcomes? First, we must accept that not all care is of equal quality: some care for patients with rheumatic diseases is bad and should be identified as such. Second, to identify good and bad quality of care, we must establish a set of quality measures. Identification of a minimum acceptable standard of evidence-based care is much easier than identifying high quality care; this has been done for some areas of rheumatology, but work needs to continue for many conditions. Third, the measures need to be evaluated against patient outcomes whenever possible. Such studies can be time-consuming and resource intensive, and sometimes they might be infeasible.

The field of quality measures is maturing and we must foster its development. Although there is the possibility for its cause to be undermined by insurance concerns, if we can assure the link between quality measures and patient outcomes, these measures will ultimately benefit our patients-a goal worthy of pursuit. 